\title{
The development and research of video on demand system based on Streaming Media Technology
}

\author{
Yuan Bin \\ Information and Education Technology Center, \\ Qujing Normal University, Qujing Yunnan, 655011 \\ Yuanbin655011@126.com
}

Keywords: Streaming media; The teaching resource; Video on demand

\begin{abstract}
With the continuous progress of the construction of digital campus, the learning resources are no longer limited to text, images and other static form, students prefer visual and audio dynamic multimedia resources, using streaming media technology to develop teaching resources VOD system, provide teaching required for video and audio data for teachers and students, and gradually improve the utilization of network video teaching digital multimedia network resources, to promote the education and teaching resources.
\end{abstract}

\section{The problems existing in the teaching resource construction}

\subsection{The scarce teaching resource repository}

Teaching resource database of resources to a large number of electronic teaching materials, PPT, Word format text data is given priority to, needs less audio and video of students' autonomous learning, students' learning activities of limited teaching resources.

\subsection{Repository of teaching resource management does not reach the designated position}

Teaching database usually adopt unified and centralized management mode, only pay attention to the secure storage of the teaching resources, lead to teaching database failed to achieve the maximum of sharing, and unreasonable on the management of teaching resources, make teaching resources when be synchronized access problems, causes the damage of resource data.

\subsection{The teaching resource construction quality is not high}

Colleges and universities in the construction of teaching resource, too pursuit of quantity and ignore the quality of resources, the teaching resource construction before demand analysis and research, teaching resource allocation imbalance, severely reduces the quality of the teaching resource construction.

\section{Streaming media technology research}

\subsection{Streaming media}

Streaming media is a new kind of network transmission technology, sequentially on the Internet in real time transmission and playing audio/visual multimedia data stream, such as streaming media technology includes data gathering, streaming media/audio codec, storage, transmission, broadcast and other fields. Is the key technologies to implement streaming media streaming, the entire audio and video and $3 \mathrm{~d}$ media such as multimedia files after compression in a certain way parsed into a compressed package, by the video server to the user's computer real-time transmission and real-time playback.

\subsection{Mainstream format of streaming media}

When using streaming media technology, audio and video files to adopt the corresponding format, different formats of files need to use different player software to play, the so-called "a key open a lock. There are three kinds of mainstream streaming Media format: the Internet are Real Networks company Real Media; Microsoft's Windows Media; Apple's QuickTime. The three companies all streaming format type as shown in table 1-1: 


\begin{tabular}{|c|c|}
\hline company & file format \\
\hline Real Networks & RA、RM、RMVB、RP、RT \\
\hline Microsoft & AVI、ASF、WMV \\
\hline Apple & MOV (Quick Time Movie ) \\
\hline
\end{tabular}

Table 1-1 Three companies mainstream streaming media format type

\subsection{Streaming media transmission protocol}

\subsubsection{Real time transport protocol and real time transmission control protocol RTP}

Real-time transport protocol (RTP) is a kind of used to transport protocol for multimedia data flow on the network, the traffic status for one-to-one, one-to-many, can provide information in time and realize flow synchronization. Not as an independent network layer, it is part of the application, usually use the UDP protocol to transmit data, with its multiple nodes and validation service implementation itself functions. Although RTP protocol provides the related function of transport layer protocols, but RTP, but with other underlying TCP, such as ATM network transmission protocol are used together, or if the underlying network provide multicast, RTP protocol can rely on transport table to transmit data to multiple destinations ${ }^{[1]}$.

\subsubsection{Real-time streaming protocol (RTSP)}

Real - Time Streaming Protocol consists of Real Networks and Netscape co-sponsored a Protocol, which defines a one-to-many application how to effectively through IP network multimedia data transmission, its system structure is located in the RTP and RTCP, using TCP or RTP, complete the data transmission. RSTP with a request made by the client and the server responds than one-way HTTP, RTP is multimedia data transmission, transfer HTML and HTTP and RTSP is a two-way street, when using the client and the server can request, and HTTP request is made by the client, the server responds, and RTSP manipulate playback, fast forward, fast down, suspend command methods ${ }^{[2]}$.

\subsubsection{Resource reservation protocol(RSVP)}

RSVP(Rresource Reserve Protocol), Due to the audio and video data flow more sensitive than traditional data to the network time delay, to high quality audio and video information transmission through the network, besides bandwidth requirement, still need other more conditions. RSVP is the resource reservation protocol on the Internet, use an RSVP reserved part of the network resources (bandwidth) measures, can to a certain extent, to provide QOS for streaming media transmission, some experimental system, such as: network video conference tools VIC has integrated the RSVP.

\section{Research teaching repository, video on demand system}

\subsection{Key techniques for video on demand}

VOD, Video On Demand, can be free to choose the content of the video program interactive video-on-demand system, its essence is the information of the users according to their own requirements for multimedia information actively, the two-way video and audio information system realizes the according to user needs to play the function of video and audio programs. VOD technology contains the computer application technology, network communication technology, multimedia technology, video processing technology, and image processing technology such as multidisciplinary and multi-field fusion of cross product, mainly covers the multimedia data compression technology, multimedia data transmission technology. Multimedia data compression technology is the core of the multimedia technology and its main research goal is data compression ratio, compression and decompression speed ${ }^{[3]}$. In the process of sound, video, data compression, the higher the compression ratio, unzip the file after the quality is poor, therefore, the need to study to solve problems is how to in the case of high compression ratio, at the same time guarantee the high quality video images. Multimedia data transmission technology mainly includes the unicast and multicast technology and radio technology.

\subsection{Video on demand system structure design}

Teaching database video on demand system based on streaming media technology, the most 
widely used three layer architecture design: the user interaction layer, layer, resource layer, each layer functions.

User layer on the one hand, is responsible for displaying information, input information, and information to determine and user interaction function, on the other hand also real-time communication and interaction.

Interactive layer mainly realize the business logic of the site, and real-time communication and user layer and resource layer.

Resource layer mainly realizes the website database access, and real-time communication and interaction. System design diagrams are shown in figure 1-1 below:

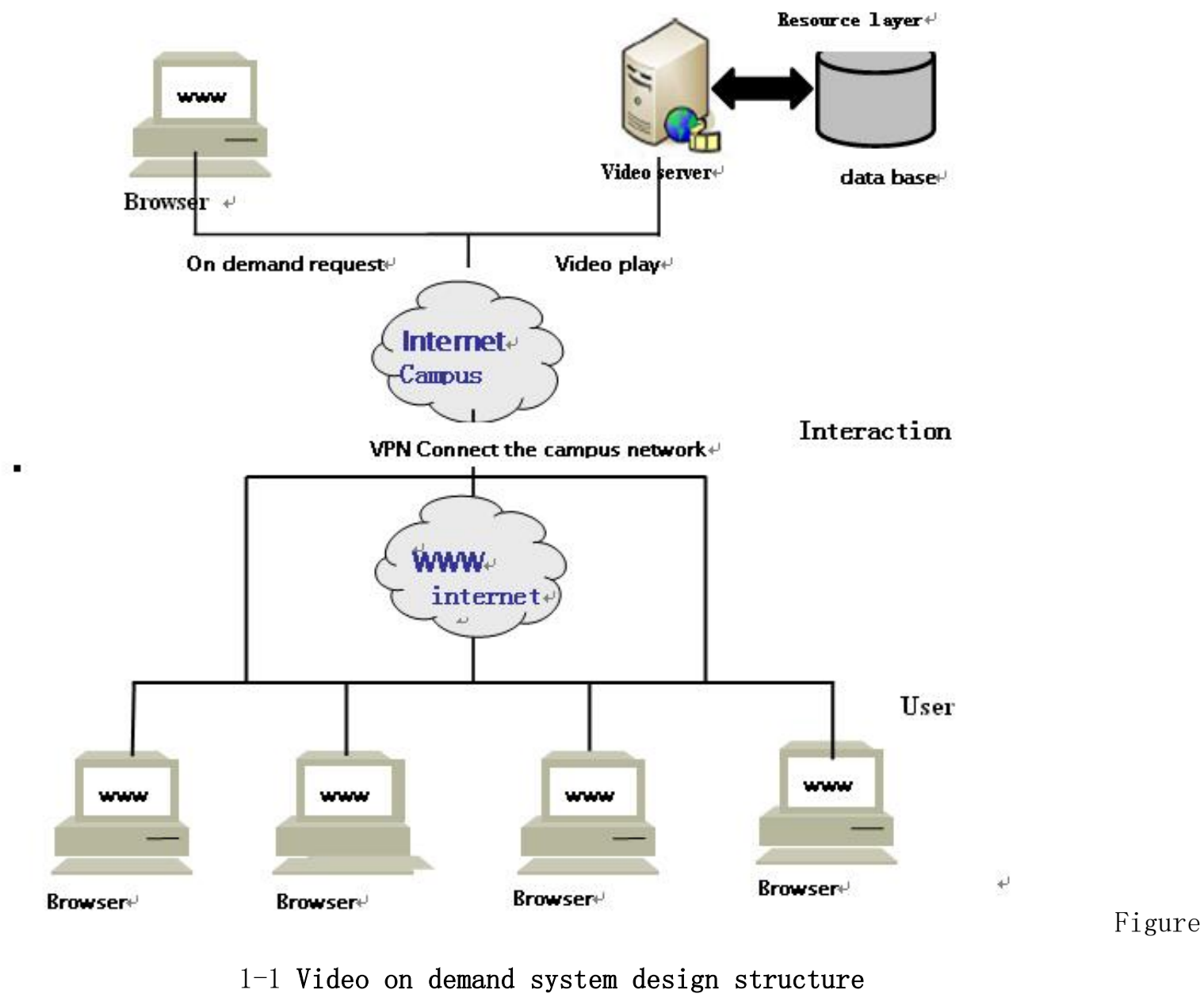

\subsection{Video on demand system design}

Teaching database video on demand system adopts B/S structure, realize the online video on demand, video retrieval, and other functions. Based on the streaming video on demand system, its purpose is to take advantage of the campus network bandwidth, realize at various levels and of different professional courses teaching online real-time video on demand, rapid retrieval of video, and other functions. Users can through the network directly connected teaching database server video on demand system, school teachers and students users can through the VPN software to connect to the campus network first, and then connect to the campus teaching database video server, so as to realize the teaching resource database of video on demand system access ${ }^{[4]}$. Among them:

(1)The teachers and students users through the network port inside the campus network can directly access database video on demand system of teaching; System administrators in order to improve the efficiency of management, can login the system to senior management system database backend server Settings, including video management, channel management, user rights management, etc.;

(2) Client on-demand video database, use Flash Player Player network real-time online 
streaming video;

(3)The server-side includes Web pages, the repository and streaming media player, the three common on demand system of interaction, communication task.

\section{Construction of teaching resource database video on demand system}

\subsection{Video on demand system function}

Within the campus network construction of video on demand system based on network platform, for the vast number of teachers and students provide is given priority to with streaming video broadcast, on-demand service, to the classroom teaching, campus activities, such as network on demand, realize the effective use of video resources, expand the way to learn. Through the video on demand system implement the following functions: video storage course teaching; Publicity of the campus news, all kinds of recreational activity, knowledge contest on demand; Network classroom and the classroom teaching video show, distance education and teaching training, etc.; The campus network video on demand learning process follow up and teaching evaluation.

\subsection{Web video on demand system function module}

Video on demand system home page modules: homepage design clear theme, form of novel, unique, creative, provide video classification list and fast retrieval channel, in the obvious position to set up the latest video, popular video recommended list; : list of video retrieval module can realize fuzzy search, keyword search, title search, classification and retrieval approaches such as source, time, search, video search, and to provide "and" and "or" search, provide video thumbnail preview mode, and provide the name of the related properties of video, video and video introduction. Video browsing module: providing video playback window, at the same time provide a playlist page, video introduction, the author of the video, and other information, if it is a teaching video also need to provide relevant teaching courseware, handouts, teaching outline and related links to address ${ }^{[5]}$.

\subsection{Video on demand system management function module}

Video transcoding processing module: vod video using h. 264 coding MP4 format, processing transcoding module using FFMPEG with Mencoder transcoding technology, implement automatic batch import video transcoding and key frames, a thumbnail generation, to video cataloging of video processing completed, add video related properties; Video upload audit module: a batch upload, breakpoint continuingly, etc; User management module: to create a system user groups and users of the system, and set up the user permissions and user quota management module, user level must be set for every user in the user group, to determine whether the user to access data media files have retrieval, on demand, download privileges; Storage management module: on demand system design for multistage storage hardware environment, multistage storage management support system work mode, support distributed storage, including the DAS and NAS storage devices ${ }^{[6]}$.

\section{System development technology research}

\subsection{Flash Media Server}

FMS (Flash Media Server) Is used for the user to communicate with each other between the new technology, it integrates the Flash multimedia interactive features, and increase the real-time voice, video, and new features such as real-time data flow. Teachers can put the recorded video, before class in FMS, and through the integration of FMS application function and communication function, through the Flash Player to provide users with the sound, video sharing and Shared data flow, FMS communication between client and server USES the RTMP real-time message transfer protocol,RTMP(Real Time Messaging Protocol) Is Adobe Systems for Flash player and audio, video and data transmission between server development open protocols, this protocol based on TCP protocol and HTTP protocol, is a specialized in the client and the server and efficient visual, audio and data of TCP/IP protocol ${ }^{[7]}$. RTMP USES port 1935 by default, the data can be a SWF format of the data, can also be a FLV format of sound and video data, brings more stable transmission effect for the user. 


\subsection{ASP.NET Dynamic web development technology}

ASP.NET Dynamic web development technology is the latest Web programming technology at present, is currently the mainstream application technology, ASP.NET is not only ASP pages and the successor is a unified Web development platform, the technology is a programming framework built on the common language runtime, can be used to produce a powerful Web application on the server end, and provides a.NET Framework framework as the basis for a new WEB development program. Compared with the traditional way and mode of Web development, ASP.NET dynamic web pages are more powerful, more flexible and easy to use, and have the advantages of more manageable and easy to expand.

\section{Conclusion}

Given the lack of teaching resource repository, resource management does not reach the designated position, construction quality is not high, and video teaching resources serious shortage of the status quo, based on "learning" as the center of the design theory as the instruction, the architecture of teaching database video on demand system based on streaming media technology, has realized the students to preview before class, after class to consolidate autonomy learning goals, expand the space of students' autonomous learning.

\section{Acknowledgement}

This paper is supported by the Science Research Foundation of Qujing Normal University (2012QN025)

\section{References}

[1] Tan Bin ,Yuan Bo. Analysis and application of streaming media technology [J]. Computer learning,2007(04):16.

[2] Li hua, Ceng Ke,Dai qionghai, etc. Streaming media technology extraction [J]. Journal of TV art, 2002(7):63-65.

[3] Liu Zhang.VOD video key technology [J].Journal of yunmeng,2008(7):147-148

[4] Kingle, cao sin, Zhou Shuyi SiWei. Streaming media technology and its application in campus network video database [J]. Journal of modern education technology,2004,(6):21-23.

[5] Chen Dai-wu,Peng Yu-xing. Streaming media technology and its application in campus education information resources transmission [J]. Journal of audio-visual education research. 2003,(9):58-61.

[6] Cheng Ming. The realization of the video on demand system in the campus network [J]. computer education,2008(22):83-84.

[7] He Qiange. Streaming media technology and its application in the courseware on demand system [J]. Computer and information technology, 2006(2): 56-58. 